\title{
TME-Responsive Multistage Nanoplatform for siRNA Delivery and Effective Cancer Therapy
}

\author{
Shuwen $\mathrm{Cao}^{1,2}$ \\ Chunhao Lin ${ }^{1,2}$ \\ Xiuling $\mathrm{Li}^{1,2}$ \\ Yixia Liang ${ }^{1,2}$ \\ Phei Er Saw $\mathbb{D}^{1,2}$ \\ 'Guangdong Provincial Key Laboratory of \\ Malignant Tumor Epigenetics and Gene \\ Regulation, Sun Yat-sen Memorial \\ Hospital, Sun Yat-sen University, \\ Guangzhou, People's Republic of China; \\ ${ }^{2}$ RNA Biomedical Institute, Sun Yat-sen \\ Memorial Hospital, Sun Yat-sen \\ University, Guangzhou, People's Republic \\ of China
}

\begin{abstract}
Since the discovery of RNA interference (RNAi), RNAi technology has rapidly developed into an efficient tool for post-transcriptional gene silencing, which has been widely used for clinical or preclinical treatment of various diseases including cancer. Small interfering RNA (siRNA) is the effector molecule of RNAi technology. However, as polyanionic macromolecules, naked siRNAs have a short circulatory half-life $(<15 \mathrm{~min})$ and is rapidly cleared by renal filtration, which greatly hinders their clinical application. Furthermore, the anionic and macromolecular characteristics of naked siRNAs impede their readiness to cross the cell membrane and therefore delivery vehicles are required to facilitate the cellular uptake and cytosolic delivery of naked siRNAs. In the past decade, numerous nanoparticles (NPs) such as liposomes have been employed for in vivo siRNA delivery, which have achieved favorable therapeutic outcomes in clinical disease treatment. In particular, because tumor microenvironment (TME) or tumor cells show several distinguishing biological/endogenous factors (eg, $\mathrm{pH}$, enzymes, redox, and hypoxia) compared to normal tissues or cells, much attention has recently paid to design and construct TMEresponsive NPs for multistaged siRNA delivery, which can respond to biological stimuli to achieve efficient in vivo gene silencing and better anticancer effect. In this review, we summarize recent advances in TME-responsive siRNA delivery systems, especially multistage delivery NPs, and discuss their design principles, functions, effects, and prospects.
\end{abstract}

Keywords: nanoparticles, siRNA, TME-responsive, multistage delivery, cancer therapy

\section{Introduction}

Since the discovery of RNA interference (RNAi) by Fire et al, ${ }^{1}$ RNAi technology has been rapidly developed into a powerful tool to clarify the body pathogenesis, and widely used for the clinical or preclinical treatment of various diseases such as malignant tumors, Hepatitis B, obesity, and multiple neuropathy. Small interfering RNA (siRNA) is an effector molecule of RNAi technology with a length of 21-23 nt. $^{2}$ Once the synthetic siRNA or double-strand RNA (dsRNAs) cleaved by enzyme Dicer enters the cytoplasm, it can bind to a protein complex called RNA-induced silencing complex (RISC). Subsequently, Argonaute 2 (Ago2), a multifunctional protein within the RISC, can unwind the siRNA double strand and the sense strand of the siRNA is cleaved. The antisense strand in the activated RISC selectively targets mRNA via complementary base pairing to achieve the enzymatic cleavage of target mRNA and gene silencing (Figure 1). ${ }^{3}$

As the activated RISC can repeatedly silence the target mRNA, the therapeutic effect usually last for several days in dividing cells and several weeks in nondividing cells. ${ }^{4}$ In addition, siRNA inhibits mRNA translation but not transcription.
Correspondence: Phei Er Saw

Email caipeie@mail.sysu.edu.cn 


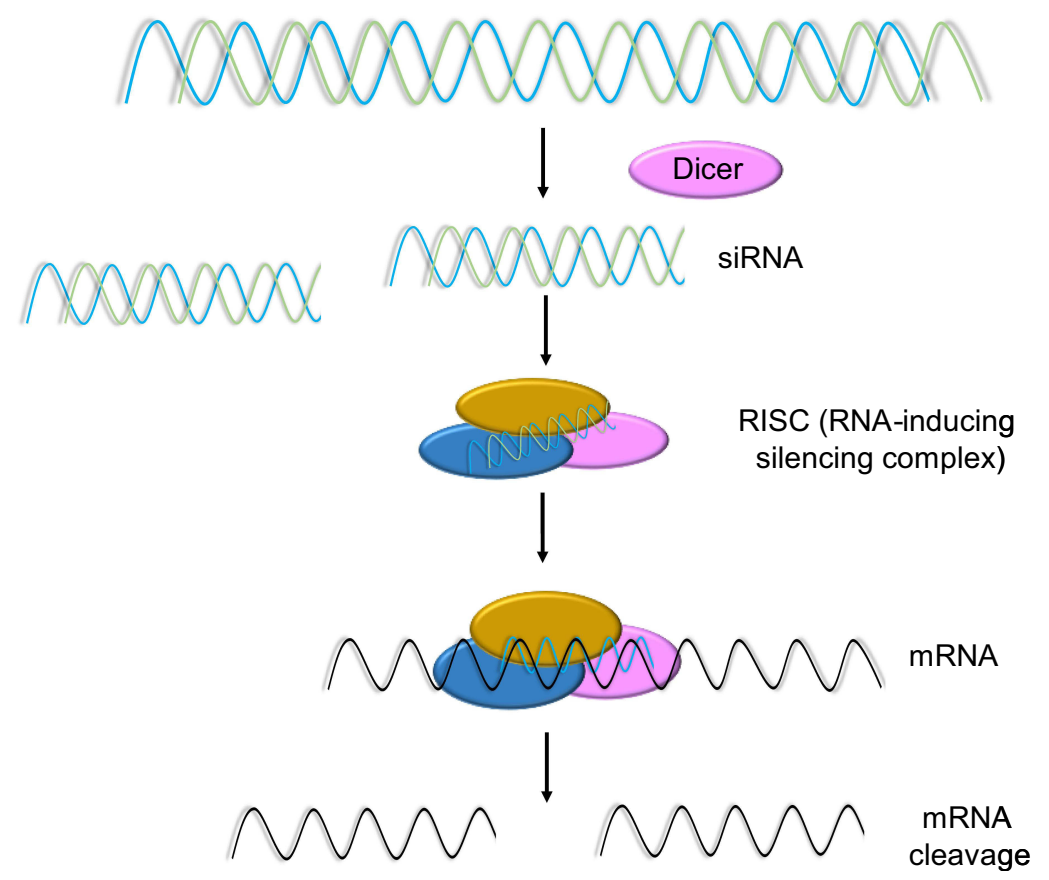

Figure I Overview of RNAi mechanism in the cell. dsRNA was processed by DICER to become short interfering RNA (siRNA), before being recruited into RISC. The complementary strand of siRNA will find complementary mRNA sequence. Once found, the ROSC complex would cleave the mRNA.

Therefore, it does not affect DNA functions and can avoid possible genetic mutations when using plasmid DNA as therapeutics. ${ }^{3}$ More importantly, siRNA can target and inhibit the translation of any disease-associated mRNAs, it therefore shows great potential for the treatment of various diseases, especially the genes that encode "undruggable" proteins.

In the past decade, tremendous investment in RNAi technology has been made by the governments worldwide. In parallel, most pharmaceutical companies have also paid much attention to develop siRNA drugs. In 2018, the US Food and Drug Administration (FDA) approved the first siRNA drug, Onpattro (Patisiran) developed by Alnylam for the treatment of Hereditary transthyretin amyloidosis (hATTR) (Trial 1/NCT01960348). Currently, another siRNA drug Inclisiran is undergoing a Phase II clinical trial for patients at high cardiovascular risk with elevated LDL cholesterol. In addition, there are around 30 siRNA drugs under clinical trial. ${ }^{5}$ However, most of these siRNA drugs focus on the treatment of liver diseases and the clinical translation of siRNA drugs for cancer therapy is still far from expected. The key challenge is to effectively in vivo transport siRNA to tumor cells. Naked siRNA has a short circulatory half-life $(<15 \mathrm{~min})$ and is easily degraded by nucleases and filtered by glomeruli. ${ }^{6}$ In addition, due to its polyanionic and macromolecular characteristics $(\mathrm{Mw} \sim 13$
$\mathrm{kDa}$ ), naked siRNA cannot deeply penetrate the tumor tissues and readily cross the cell membrane. ${ }^{7,8}$ Nanoparticles (NPs) such as liposomes and polymeric NPs exhibited unique advantages in tumor targeting, transmembrane delivery and tumor growth suppression. ${ }^{9,10}$ Introducing NPs to encapsulate siRNA can protect it from degradation and efficiently deliver siRNA to tumor cells, NPs-mediated delivery systems have been widely used for in vivo gene silencing and cancer therapy. In particular, because tumor tissues or tumor cells show different microenvironment (eg, acidic $\mathrm{pH}$, overexpression of enzymes, hypoxia) compared to normal tissues or cells, much attention has recently paid to develop Tumor microenvironment (TME)-responsive NPs, which can respond to biological stimuli to achieve efficient in vivo gene silencing and better anticancer effect. ${ }^{11-13}$ In this review, we comprehensively summarize the recent development of TME-responsive siRNA delivery systems and discuss their application prospect.

\section{NPs-Mediated siRNA Delivery}

Viral vectors are the earliest carrier used for in vivo siRNA delivery. However, viral vectors show the drawbacks of potential immunogenicity, mutagenicity, and difficulty in scale-up. ${ }^{14}$ In the past decade, non-viral vectors, especially lipid and polymeric NPs, have been widely used as alternative carriers, due to their characteristics of good biocompatibility, low 
immunogenicity and ease in scale-up. ${ }^{15,16}$ The representative example is the siRNA drug Onpattro, which is essentially a liposome nanoplatform with therapeutic siRNA encapsulated into its nanostructure. When using NPs to deliver siRNA for cancer therapy, they can passively target to the tumor sites via the enhanced permeability and retention (EPR) effect, which thus facilitates to improve the tumor siRNA accumulation in tumor tissues and enhance its gene silencing effect. ${ }^{17}$ However, increasing evidence have demonstrated that the traditional NPs cannot completely overcome the multiple physiological barriers in the tumor tissues during in vivo siRNA delivery (Figure 2).

Therefore, an ideal in vivo siRNA delivery nanoplatform should have the following features: i) good biocompatibility and low systematic immune response; ii) long blood circulation to protect siRNA from clearance and degradation; iii) high accumulation in the tumor sites and deep penetration into the tumor tissue; iv) higher uptake by tumor cells and efficient release of loaded siRNA in the cytoplasm to exert its biological function. To achieve this goal, the researchers have recently taken advantage of the distinguishing microenvironment in tumor tissue or tumor cell, and have utilized series of chemical strategies to design TME-responsive NPs to improve the in vivo siRNA delivery efficacy and enhance gene silencing effect.

\section{TME-Responsive NPs-Mediated Multistage siRNA Delivery}

Solid tumor is a systemic disease and its growth relies on the surrounding microenvironment. ${ }^{18}$ Besides the highly heterogeneous tumor cells, solid tumor also contains extracellular matrix, fibroblast, blood vessel/lymphatic, and immune cells, which together constitute the tumor microenvironment. ${ }^{19}$ The interaction between tumor microenvironment and tumor cells plays an important role in tumor genesis, progression and metastasis. Tumor cells act to the microenvironment by secreting growth factors and proteases, and the acclimated microenvironment cells feed back to the tumor cells. ${ }^{20}$ This interaction makes the tumor tissues showing different physiological microenvironment from normal tissues, such as weakly acidic and hypoxic microenvironment. Based on this distinguishing microenvironment, designing TME-responsive NPs could address the issues encountered in the siRNA delivery by traditional NPs. These TME-responsive NPs are stable during blood circulation and in the normal tissues. After arrival to the tumor sites, siRNA is rapidly released in the tumor tissue or tumor cells, which could significantly improve the bioavailability of siRNA and enhance the anti-tumor effect. At present, the reported TME-responsive NPs mainly include pH-, hypoxia-, enzyme-, reactive oxygen species (ROS)-, and

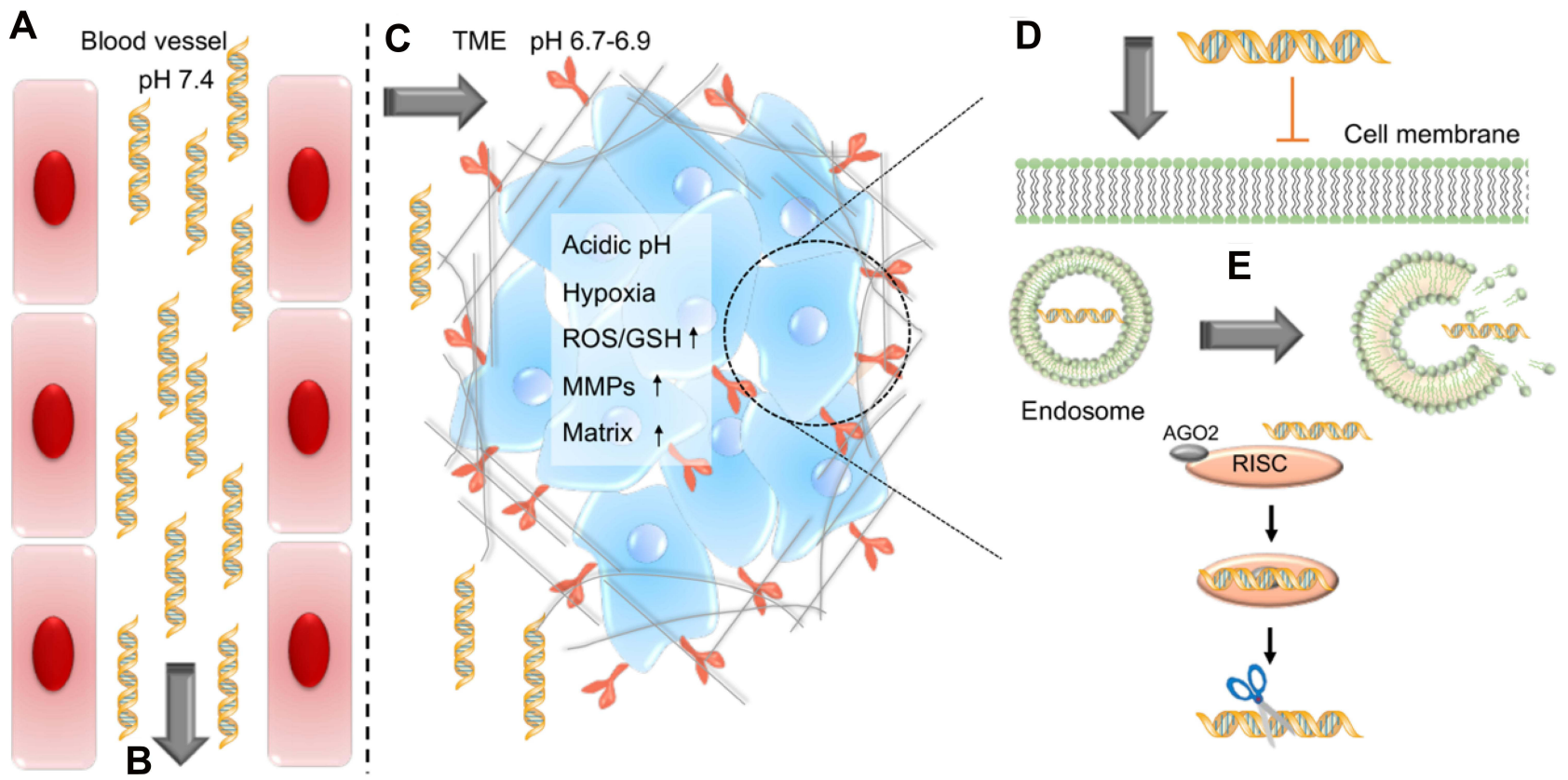

Figure 2 Major barriers in siRNA therapeutics. (A) siRNA is degraded within seconds in light of the abundance of RNases in circulation. (B) The short half-life of naked siRNA warrants a capable delivery carrier that could safely and effectively deliver siRNA to TME. (C) TME exhibited unique characteristics, including acidic pH, hypoxia, increased redox stress, elevated enzyme expression and abundant extra cellular matrix, which further impede the delivery of siRNA. (D) Even when siRNA is successfully delivered to the tumor site, being an anionic biomacromolecule, siRNA is unable to easily penetrate the negatively charged cell membrane. (E) After cellular uptake, siRNAs need to escape endosomes to exert its gene silencing mechanism in the cell cytoplasm. 
reduction-responsive NPs (Table 1). The applications of these TME-responsive NPs for in vivo siRNA delivery are described in the following sections.

\section{Targetable Attributes of the TME}

\section{The Acidic pH}

As compared to the $\mathrm{pH}$ levels of normal tissue $(\sim \mathrm{pH} 7.2-7.5)$, $\mathrm{pH}$ levels within the TME are usually more acidic $(\sim \mathrm{pH} 6.5-$ $6.9)^{31}$ because of the increased metabolic rate of cancer cells to support their high energy requirements for survival. ${ }^{31,32}$
The Warburg effect describes the tumor's acidic environment as a consequence of glycolysis (the conversion of glucose into lactic acid). ${ }^{33}$ By utilizing $\mathrm{pH}$-responsive polymers, ${ }^{34}$ acid-labile chemical bonds ${ }^{35,36}$ or ionizable chemical groups, nanocarriers can be designed to be $\mathrm{pH}$-sensitive.

\section{Upregulation of Enzymes}

Another important target in the TME is the upregulation of enzymes from tumor cells and tumor stromal cells. ${ }^{37,38}$ Certain enzymes that are over-expressed by solid tumors

Table I siRNA-Based Drugs for Solid Tumor Therapy and Their Current Status in Clinical Trials

\begin{tabular}{|c|c|c|c|c|c|c|c|}
\hline Drug & $\begin{array}{l}\text { Method of } \\
\text { Delivery }\end{array}$ & Disease & $\begin{array}{l}\text { Clinical Trial } \\
\text { No. }\end{array}$ & Phase & Status & $\begin{array}{l}\text { Year } \\
\text { Completed }\end{array}$ & Ref \\
\hline \multirow{2}{*}{$\begin{array}{l}\text { ALN- } \\
\text { VSPO2 } \\
\text { Alnylam } \\
\text { Target: } \\
\text { KSP and } \\
\text { VEGF }\end{array}$} & \multirow[t]{2}{*}{ Intravenous/LNP } & \multirow[t]{2}{*}{ Solid Tumors } & $\begin{array}{l}\text { NCT } \\
00882180\end{array}$ & $\begin{array}{l}\text { Phase } \\
\text { I }\end{array}$ & Completed & 2011 & \multirow[t]{2}{*}[21,22]{} \\
\hline & & & NCT0II 58079 & $\begin{array}{l}\text { Phase } \\
\text { I }\end{array}$ & Completed & 2012 & \\
\hline $\begin{array}{l}\text { CALAA-0I } \\
\text { Calandro } \\
\text { Target: } \\
\text { RRM2 }\end{array}$ & $\begin{array}{l}\text { Intravenous/ } \\
\text { Cyclodextrin } \\
\text { polymer-based } \\
\text { nanoparticles }\end{array}$ & Solid Tumors, Cancer & $\begin{array}{l}\text { NCT } \\
00689065\end{array}$ & $\begin{array}{l}\text { Phase } \\
\text { I }\end{array}$ & Terminated & 2012 & {$[23,24]$} \\
\hline \multirow{2}{*}{$\begin{array}{l}\text { SIGI2D } \\
\text { Silenseed } \\
\text { Target: } \\
\text { KRAS }\end{array}$} & \multirow[t]{2}{*}{$\begin{array}{l}\text { Implant/LODER } \\
\text { polymer }\end{array}$} & \multirow[t]{2}{*}{$\begin{array}{l}\text { Pancreatic Ductal Adenocarcinoma, } \\
\text { Pancreatic Cancer }\end{array}$} & $\begin{array}{l}\text { NCT } \\
01188785\end{array}$ & $\begin{array}{l}\text { Phase } \\
\text { I }\end{array}$ & Completed & 2013 & \multirow[t]{2}{*}{ [25] } \\
\hline & & & $\begin{array}{l}\text { NCT } \\
01676259\end{array}$ & $\begin{array}{l}\text { Phase } \\
\text { II }\end{array}$ & Recruiting & Est: 2020 & \\
\hline \multirow[t]{2}{*}{$\begin{array}{l}\text { TKM-PLKI } \\
\text { Tekmira } \\
\text { Target: } \\
\text { PLK-I }\end{array}$} & \multirow[t]{2}{*}{ Intravenous/LNP } & $\begin{array}{l}\text { Colorectal Cancer with Hepatic } \\
\text { Metastases, Pancreas Cancer with Hepatic } \\
\text { Metastases, Gastric Cancer with Hepatic } \\
\text { Metastases, Breast Cancer with Hepatic } \\
\text { Metastases, Ovarian Cancer with Hepatic } \\
\text { Metastases }\end{array}$ & $\begin{array}{l}\text { NCT } \\
01437007\end{array}$ & $\begin{array}{l}\text { Phase } \\
\text { I }\end{array}$ & Completed & 2012 & {$[26,27]$} \\
\hline & & $\begin{array}{l}\text { Cancer, Neuroendocrine Tumors, NET, } \\
\text { Adrenocortical Carcinoma, ACC }\end{array}$ & $\begin{array}{l}\text { NCT } \\
01262235\end{array}$ & $\begin{array}{l}\text { Phase } \\
\text { I }\end{array}$ & Completed & 2015 & \\
\hline $\begin{array}{l}\text { DCR-MYC } \\
\text { Dicerna } \\
\text { Target: } \\
\text { MYC }\end{array}$ & $\begin{array}{l}\text { Intravenous/ } \\
\text { LNP-formulated } \\
\text { Dicer substrate } \\
\text { siRNA (DsiRNA) }\end{array}$ & $\begin{array}{l}\text { Solid Tumors, Multiple Myeloma, Non- } \\
\text { Hodgkin's Lymphoma, Pancreatic } \\
\text { Neuroendocrine Tumors, PNET, NHL }\end{array}$ & $\begin{array}{l}\text { NCT } \\
02110563\end{array}$ & $\begin{array}{l}\text { Phase } \\
\text { I }\end{array}$ & Terminated & 2016 & {$[28,29]$} \\
\hline $\begin{array}{l}\text { siRNA- } \\
\text { EphA2 MD } \\
\text { Anderson } \\
\text { Target: } \\
\text { EphA2 }\end{array}$ & Intravenous/LNP & Advanced Cancers & $\begin{array}{l}\text { NCT } \\
01591356\end{array}$ & $\begin{array}{l}\text { Phase } \\
\text { I }\end{array}$ & Recruiting & Est: 2021 & {$[30]$} \\
\hline
\end{tabular}

Abbreviations: KSP, Kinesin Spindle Protein; VEGF, Vascular Endothelial Growth Factor; RRM2, Ribonucleoside-diphosphate reductase subunit M2; PLK-I, Polo-like Kinase protein-I; KRAS, Kirsten Rat Sarcoma; EphA2, Ephrin type-A receptor 2. 
and the TME include peptidases (eg, aminopeptidase), proteases (eg, matrix metalloproteinase and cathepsin B), and lipases (eg, phospholipase A2). In high concentrations, these enzymes serve a crucial role in the growth of tumor cells, angiogenesis, invasion, and metastasis, which serves as a key stimulus for a responsive nanoparticle system.

\section{Hypoxia}

The abnormal vasculature in solid tumors is unable to provide adequate oxygenation to the entire tumor tissue (especially to the inner regions with poor vascularization). In addition, the consumption of oxygen is further accelerated by the over-proliferation of tumor cells. ${ }^{39}$ Hypoxia in the TME is mainly due to these two factors. Evidently, the hypoxic regions of the TME that are distant from blood vessels are unfavorable to large nanoparticles which possess low penetration ability into deep tissue. Hence, systems of hypoxia-sensitive nanoparticles have been developed, and can be classified into two major mechanisms. The first mechanism describes nitroimidazole-based micelle systems that are bio-reduced to aminoimidazole, creating a hydrophobic-to-hydrophilic transition, which then triggers micelle disassembly. ${ }^{40}$ The second mechanism entails the use of azobenzene group-employed siRNA nanocarriers which are cleavable, leaving the polyethylene imine (PEI/siRNA) complex with a positively charged surface for the facilitation of cellular uptake. ${ }^{41}$

\section{Elevated Redox Stress (ROS/GSH)}

Due to the elevated levels of superoxide anion radicals, hydrogen peroxide and hydroxyl radicals in the TME, tumor cells experience an increased potential of oxidative stress. $^{42}$ As a result, tumor cells will generally upregulate the reduction potential by the expression of redox species such as glutathione (GSH) and superoxide dismutase (SOD) to overcome the oxidative stress. Consequently, upregulated redox levels of the tumor create a high overall potential (oxidative/reductive) in the TME. This dysregulated potentials of oxidation and reduction in the TME position them as excellent candidates for the development of TME-targeted nanoparticles. Furthermore, the level of reactive oxygen species (ROS) in cancer cells is higher compared to normal cells due to aerobic metabolism as a result of oncogenic transformation ${ }^{43}$ This is evidenced by recent studies that described the constant generation of high levels of intracellular ROS by cancer cells (ie, hydrogen peroxide, hydroxyl radicals, and superoxide) as compared to normal cells, due to oncogenic transformation. ${ }^{44}$
Prospectively, the design and application of a novel siRNA delivery system which will respond to different pathophysiological properties in the TME, such as increased or unique levels of functional proteins, amino acids, and DNA fragments, will be available for tumor targeting. ${ }^{45}$ As a whole, multi-responsive NPs can exploit the various targets present in the TME as compared to traditional NPs, as they are able to be activated at the target tumor site by the triggering of the unique characteristics present in the TME, as discussed above. The cargoes can then be released at a higher concentration, and ondemand, to the tumor site. ${ }^{33,36}$

\section{Development of Bioresponsive Multistage siRNA Targeting Strategy Concept of Multistage-Responsive siRNA Nanocarrier}

Conventional nanocarriers can be modified to be responsive and sensitive to the unique stimuli present in the TME. These carriers will receive the necessary stimulation which alters the physicochemical structures or features when it reaches the TME, consequently realizing their desired functions. In the following sections, multiple responsive modes of multistage responsive siRNA nanocarriers and their associated functions are discussed. Various TME stimuli, especially the upregulated enzymes and acidic $\mathrm{pH}$, can activate approximately similar behaviors of these carriers in tumor tissue. In general, the responsive modes in the TME include targeting ligand exposure (re-emergence of targeting ligand), polyethylene glycol (PEG) detachment, particle-size shrinkage, and surface charge reversal. ${ }^{36}$

As contrasted to drug delivery system (DDS), additional cargo preservation and endosomal release capabilities are required by siRNA delivery vehicles. If discharged extracellularly or in close proximity to tumors, small molecule drugs could still undergo passive diffusion through the cell membrane and be situated in the cell cytoplasm to carry out its functions. Due to charge repulsion, siRNAs, which are anionic biomacromolecules, cannot penetrate the cell membrane without aid. Hence, it is crucial to ensure the safe and efficient delivery of siRNAs across the cell membrane. ${ }^{46}$ Upon safe transportation of siRNAs into the cell via nanocarriers, it must avoid endosomes to execute its potent mRNA degradation machinery. For these reasons, a "multi-stage delivery approach" is necessary for the development of an effective siRNA 
delivery system, which guarantees the systemic delivery of siRNA. ${ }^{47}$ Herein, we summarized the examples which fulfilled all three stages of the multistage siRNA delivery system (Table 2).

\section{Multi-Stage Responsive Nanocarrier for siRNA Delivery}

To achieve both stability in the circulation as well as rapid release of siRNAs in the TME, a number of nanocarriers were designed based on the abnormal TME stimuli such as a slightly acidic $\mathrm{PH}$, elevated enzymes, hypoxia and increased ROS. Herein, we highlighted some designs of multi-stage responsive nanocarriers.

\section{TME pH-Responsive Nanocarriers}

Yang et $\mathrm{al}^{48}$ designed a rapid $\mathrm{pH}$-responsive sheddable nanoparticle (S-NP), which was prepared as follows. PEGylated tumor acidity-responsive anionic polymer PPC-DA was introduced to the surface of positively charged ssPEI800/siRNA nanoparticles by electrostatic interaction. To obtain PPC-DA, the diblock polymer $\mathrm{mPEG}_{45}$-b-PAEP ${ }_{75}$ was first synthesized. Then, the $\mathrm{mPEG}_{45}$-b- $\mathrm{PAEP}_{75}$-Cya (PPC) was synthesized via the thiol-ene "click" reaction of $\mathrm{mPEG}_{45}-\mathrm{b}-\mathrm{PAEP}_{75}$ with cysteamine. The PPC was further reacted with 2,3-dimethylmaleic anhydride (DMMA) to obtain the tumor acidityresponsive PPC-DA. DMMA-modified amines are usually stable under neutral $\mathrm{pH}$, yet rapidly degrade in slightly acidic TME conditions. Upon exposure to slightly acidic $\mathrm{pH}$, the amide bond of PPC-DA in S-NP was triggered, which led to degradation and the generation of positively charged amino groups, which further led to a strong electrostatic repulsion to positively charged ssPEI800/siRNA complex, resulting in the phenomenon of "sheddable nanoparticles" (Figure 3A).

Sun et $\mathrm{al}^{49}$ created the "Acid-labile polymer" which is sensitive towards the $\mathrm{pH}$ in the TME. The Dlink $\mathrm{m}_{\mathrm{m}}$ Bridged Copolymer was constructed with PEG, Dlink $\mathrm{m}_{\mathrm{m}}$ and PLGA. Cyclohexanedimethanol (CDM) was first introduced to provide an additional reaction site to construct a bridged responsive copolymer. Also, it decelerated the linkage sensitivity to

Table 2 Multi-Stage Responsive siRNA Delivery Systems

\begin{tabular}{|c|c|c|c|c|}
\hline $\begin{array}{l}\text { STAGE I Physiological } \\
\text { Changes Upon Stimuli in } \\
\text { TME }\end{array}$ & $\begin{array}{l}\text { STAGE } 2 \text { Efficient siRNA } \\
\text { Delivery Through Cell } \\
\text { Membrane }\end{array}$ & $\begin{array}{l}\text { STAGE } 3 \text { Endosomal } \\
\text { Escape Mechanism }\end{array}$ & $\begin{array}{l}\text { STAGE } 4 \text { siRNA } \\
\text { Release Mechanism }\end{array}$ & Reference \\
\hline $\mathrm{pH}$ - sheddable PEG layer & Polycation/siRNA complex & Proton sponge effect & - & [48] \\
\hline \multirow[t]{2}{*}{$\mathrm{pH}$ - acid labile polymer } & CPP/siRNA complex & Proton sponge effect & - & {$[35,49]$} \\
\hline & PEI/siRNA complex & Proton sponge effect & - & \\
\hline $\mathrm{pH}$ - borate ester linker & PBA/siRNA complex & Proton sponge effect & & [50] \\
\hline $\mathrm{pH}$ - charge reversal polymer & TCPA peptide/siRNA complex & Proton sponge effect & - & [34] \\
\hline $\mathrm{pH}$ - charge reversal polymer & bPEl/siRNA complex & Proton sponge effect & GSH & {$[51]$} \\
\hline $\mathrm{pH}$ - charge reversal polymer & PEl/siRNA complex & Proton sponge effect & $\begin{array}{l}\text { Redox sensitive linker } \\
\text { (GSH) }\end{array}$ & {$[52]$} \\
\hline $\mathrm{pH}-\mathrm{pH}$ responsive polypeptide & Cell-penetrating peptide & & & [53] \\
\hline pH - PPy nanoparticle & $\mathrm{pH}$-triggered cleavable backbone & $\begin{array}{l}\text { Size-tunable } \mathrm{PPy} / \mathrm{pH}- \\
\text { triggered drug release }\end{array}$ & & \\
\hline MMP enzyme - sheddable PEG & PASp(DET)/siRNA complex & $\begin{array}{l}\text { PAsp(DET) is an excellent } \\
\text { endosome destabilizer }\end{array}$ & - & [54] \\
\hline $\begin{array}{l}\text { MMP enzyme - cleavable peptide } \\
\text { linker }\end{array}$ & Polycation/siRNA complex & Proton sponge effect & - & [55] \\
\hline MMP 7 cleavable peptide & DMAEMA/siRNA complex & Proton sponge effect & & {$[56,57]$} \\
\hline Hypoxia - PAPD polymer & PEI-DOPE/siRNA complex & Proton sponge effect & - & {$[41]$} \\
\hline Redox responsive & Cationic lipid/siRNA complex & Proton sponge effect & - & [58] \\
\hline
\end{tabular}



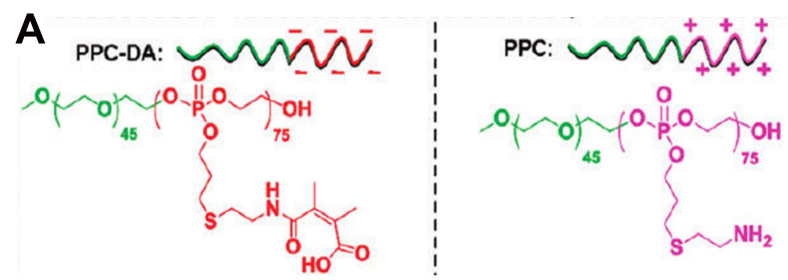

$\mathbf{B}$

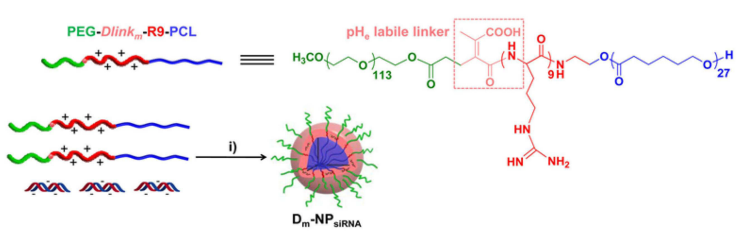

C

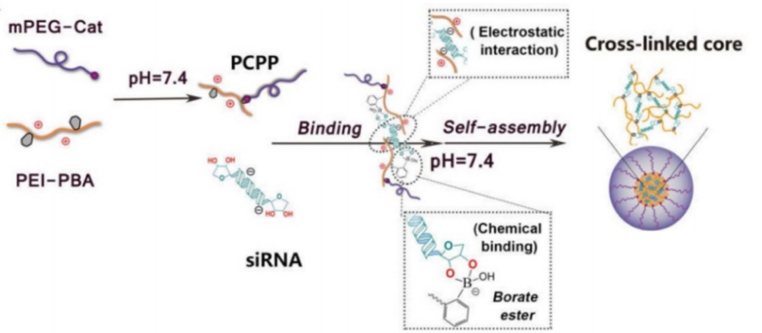

D

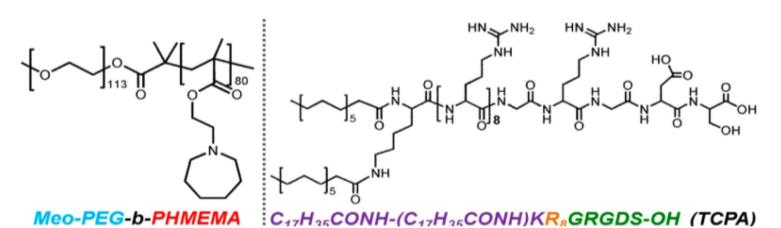

Figure 3 (A) The charge reversal properties of PPC-DA from negative charge in physiological pH to positive charge at TME pH; Reproduced from Yang XZ, Du JZ, Dou S,

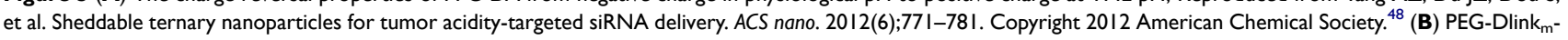
R9-PCL-based siRNA delivery system with a TME pH labile linker which could be cleaved at pH levels lower than 6.8; Reproduced from Sun CY, Shen S, Xu CF, et al. Tumor acidity-sensitive polymeric vector for active targeted siRNA delivery. J Am Chem Soc. 2015;137:15,217-15,224. Copyright 2015 American Chemical Society. ${ }^{49}$ (C) The combination of mPEG-Cat and PEI-PBA could be self-linked and displayed binding to siRNA at pH 7.4. This linkage could be broken at pH levels in the TME, releasing the siRNA complex; Reproduced with permission from Fan B, Kang L, Chen L, et al. Systemic siRNA delivery with a dual pH-responsive and tumor-targeted nanovector for inhibiting tumor growth and spontaneous metastasis in orthotopic murine model of breast carcinoma. Theranostics. 2017;7(2):357-376. IVYSPRING..$^{50}$ (D) Creation of TME PH-sensitive Meo-PEG-b-PHMEMA-based NPs, with TCPA/siRNA complex; Reproduced from Xu X, Saw PE, Tao W, et al. Tumor microenvironment-responsive multistaged nanoplatform for systemic RNAi and cancer therapy. Nano letters. 2017;170:4427-4435. Copyright 2017 American Chemical Society. ${ }^{34}$

$\mathrm{pH} 7.4$, thus satisfying the requirement of cellular uptake. After the well-established synthesis of chlorine substituted CDM, PEG was modified into PEG-DMA. Then, PEG-
DMA was coupled with an amine-functionalized poly( $\varepsilon$ caprolactone)-nonaarginine R9 (PCL-R9, Figure 4B). Under slightly acidic $\mathrm{pH}$ conditions, an increasing
A

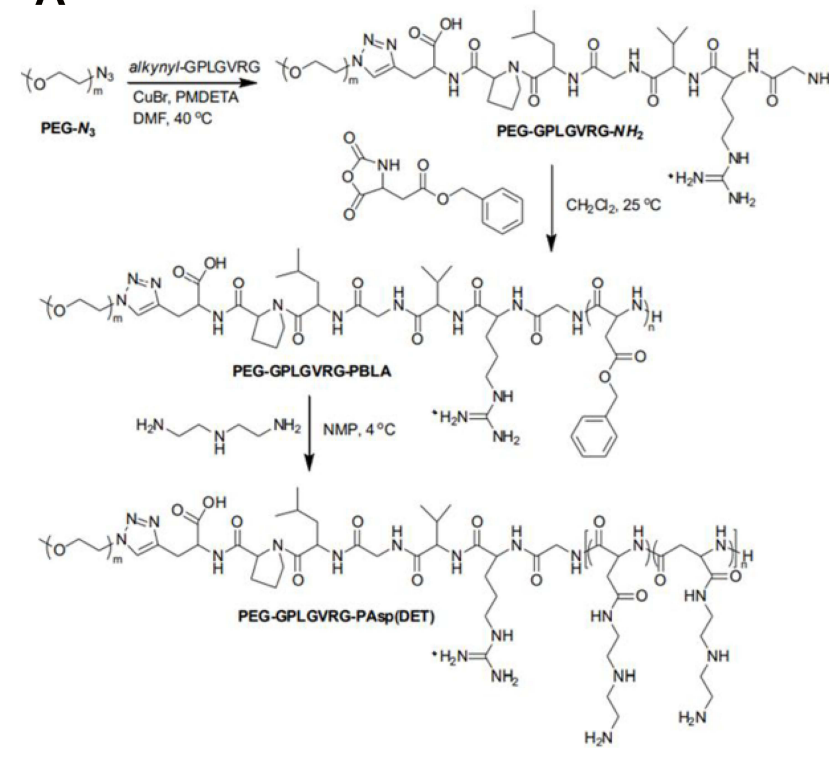

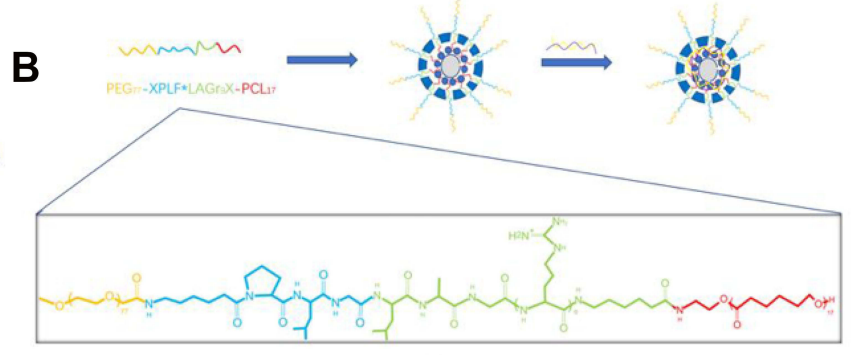

C
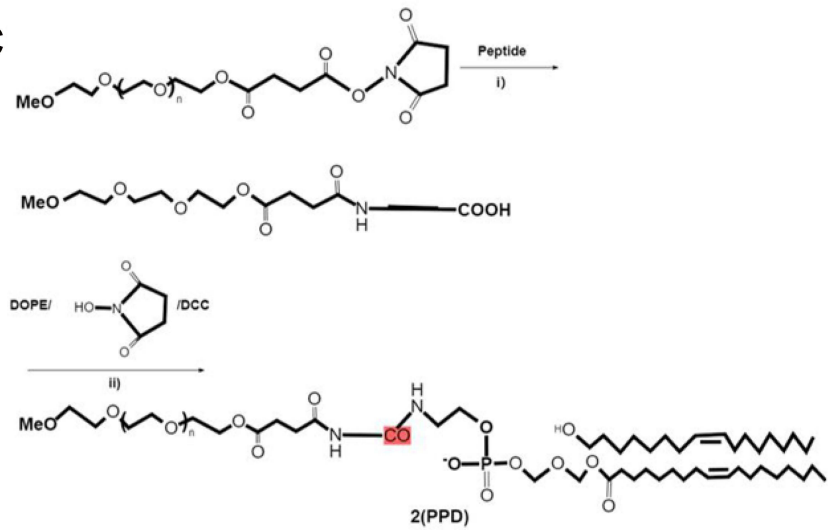

Figure 4 (A) Synthesis scheme of PEG-GPLGVRG-PAsp (DET; Reproduced from Li J, Ge Z, Liu S. PEG-sheddable polyplex micelles as smart gene carriers based on MMPcleavable peptide-linked block copolymers. Chem Commun. 201 3;49: 6974-6976, @ 2013 The Royal Society of Chemistry. ${ }^{54}$ (B) Synthesis of block copolymer of PEG and poly ( $\varepsilon-$ caprolactone) (PCL) with an MMP-2-sensitive peptide bridge, namely Pro-Leu-Gly-Leu-Ala-Gly-Arg-Arg-Arg-Arg-Arg-Arg-Arg-Arg-Arg (PLG*LAGr9). (C) Synthesis of PPDMEND (constructed with PEG/matrix metalloproteinase (MMP)-substrate peptide 20 / I,2-dioleoyl-sn-glycero-3-phosphoethanolamine (DOPE) ternary conjugate (PPD)). 
concentration of homopolymers was found with a prolonged elution volume $(9.4 \mathrm{~mL})$, which was consistent with the lower molecular weight of PEG and PCL-R9, indicating degradation of the polymer. After self-assembly, the R9 in the hydrophilic shell of Dm-NP and NP allowed them to bind with siRNA to form micelleplexes. Image analysis detected almost $\sim 85 \%$ of the intact siRNA when the nanoparticles were treated by RNase A, suggesting the feasibility of DmNP in protecting the siRNA from enzymolysis and serum degradation (Figure 3B).

Wang et $\mathrm{al}^{59}$ conjugated the cationic (PLGA) 2 -PEI with 2,3-dimethylmaleic anhydride (DMMA) that reacted with the amines in PEI to generate amide bonds. The final nanoparticles were stable and exhibited negative surface charge at neutral or alkaline conditions, but could release the DMMA molecules in a weakly acidic niche due to the prompt hydrolysis of the amide bonds. The amino groups in PEI were exposed again, resulting in the charge conversion of the nanoparticles from negative to positive. Therefore, the nanoparticles could specifically deliver siRNAs into tumor cells with high efficiency and in turn, decreased the expression of Nogo-B receptor (NgBR) in cancer cells and tumor-associated endothelial cells.

Fan et $\mathrm{al}^{50}$ developed a nanovector which consisted of $\alpha$-methoxy $\omega$-amino polyethylene glycol-catechol (mPEGCat) and PBA-grafted low molecular weight branched polyethylenimine (1800 Da) (PEI-PBA). Here, catechol (Cat) is an excellent cis diol-containing reagent to form complexes with boronic acids. The spontaneous formation of boronate linkages between PBA and Cat allows the facile attachment of mPEG-Cat onto PEI-PBA at neutral conditions. The PBA-functional polymer (PEG-CPB-PEI) could condense siRNAs into polyplex nanoparticles at $\mathrm{pH}$ 7.4 via a "synergetic assembly" approach of facile PBAribose chemical binding and an electrostatic interaction generated between the cationic PEI with the anionic phosphate group of siRNAs. After internalization into the endosomes/lysosomes, the borate ester bond of PBAribose in the cross-linked core would be completely disrupted as the $\mathrm{pH}$ value further declines to $\mathrm{pH} 4.5 \sim 5.5$. Therefore, complete breakdown of the nanostructure would occur accompanied by the rapid and triggered release of siRNAs. Meanwhile, the PEI component promotes endosomal/lysosomal escape which allows siRNAs to migrate into the cytoplasm for gene silencing (Figure 3C).

We also developed a nanoparticle which was sharply responsive to the TME-responsive nanoparticle by utilizing a unique charge-reversal polymer which could be triggered by the acidic TME $\mathrm{pH}$ to reverse the polymer charge from negative to positive. By introducing the hydrophobic poly(2-(hexamethyleneimino)ethyl methacrylate) (PHMEMA) which demonstrated a sharp response to $\mathrm{pH}$ levels in the TME, in combination with the PEG surface shell, which is a charge-mediated complex of siRNA and cell penetrating peptide, we developed an easy-tocontrol vector with high siRNA delivery efficiency triggered by $\mathrm{pH}$ change in a narrow range between $\mathrm{pH} 6.8$ and 7.4 (Figure 3D). ${ }^{34}$

Yang et $\mathrm{al}^{53}$ provided a novel delivery tactic of building a new therapeutic agent delivery system by utilizing photo- and $\mathrm{pH}$-responsive polypeptides (PPPs), and this delivery system could transport cargoes with greater selectivity and efficiency to tumor cells. In this system, the PPP consists of three elements: a cell-penetrating peptide sequence (CGRRMKWKK), a photodecomposable group (4, 5-dimethoxy-2-nitrobenzyl group) and a pH-sensitive inhibitory peptide (EEEERRRR). After systemic administration, PPP-NPs accumulated at the tumor sites due to the enhanced permeability and retention (EPR) effect. The cell penetration ability of CPPs is effectively shielded by opposite electrical charges within the $\mathrm{pH}$-sensitive inhibitory peptides in circulation. Upon Near Infra-red NIR light irradiation at the tumor location, the photo-cleavable group is cleaved. Simultaneously, the $\mathrm{pH}$-sensitive inhibitory peptide eliminates the electrostatic attraction. After cleavage of the linker and elimination of the electrostatic attraction, the PPP could release its inhibitory peptides to expose the CPPs. Then, with the help of revitalized CPPs, the NPs could efficiently enter into the cancer cells.

Tubular polypyrrole (PPy) with tunable 60-200 nm diameters was successfully synthesized using a $\mathrm{TiO}_{2}$ nanotube array template in the presence of $\mathrm{FeCl}_{3}$ and pyrrole monomer. Aromatic imine bonds were introduced into the PPy nanoparticles via reaction with tetra-arm aldehyde (TAA) to prepare a novel pH-triggered cleavable backbone. One end of PEG was functionalized with FA as the targeting ligand to bind with $\alpha$-FR on the surface of tumor cells, whereas the other end was chemically conjugated to the surface of PPy nanoparticles via a cleavable aromatic imine bond to control the release of the drug. Guided by the optimal size of PPy nanoparticles, the therapeutic efficacy of Doxorubicin-loaded PPy nanoparticles was demonstrated both in vitro and in vivo. ${ }^{60}$ 


\section{TME Enzyme-Responsive Nanocarriers}

Matrix metalloproteinases (MMPs) are a group of proteases involved in tumor invasion and are significantly upregulated in the TME. ${ }^{61}$ With this characteristic, a TME-specific targeting strategy was developed. MMPcleavable peptide was utilized as a linker to construct the backbone of nanocarriers. As a result, the nanocarrier demonstrated both stability in the bloodstream as well as rapid internalization and endosomal escape.

Li et al ${ }^{54}$ constructed PEG-sheddable polyplex micelles as smart gene delivery vectors by utilizing a matrix metalloproteinase-cleavable peptide-linked block copolymer. This nanocarrier was demonstrated to exhibit higher cellular uptake, improved endosomal escape, and highefficiency gene transfection in the presence of matrix metalloproteinase-2 (MMP-2). To achieve the goal, they utilized a MMP-2 cleavable peptide GPLGVRG to link the PEG and PAsp(DET) to synthesize the PEG-GPLGVRGpolymer backbone (Figure 4A). Upon arrival at the TME, the overexpressed MMP-2 will break the polymer backbone and remove the surface PEG layer to enhance the internalization ability of the nanocarrier. As PAsp(DET) is an excellent endosome destabilizer, the encapsulated siRNA would be able to escape from the endosome and therefore silence the target gene.

Wang et $\mathrm{al}^{62}$ reported site-specific decladding MMP-2triggered sheddable polymeric micelles for siRNA delivery. They synthesized a block copolymer of PEG and poly $(\varepsilon-$ caprolactone) (PCL) with an MMP-2-sensitive peptide bridge, namely Pro-Leu-Gly-Leu-Ala-Gly-Arg-Arg-ArgArg-Arg-Arg-Arg-Arg-Arg (PLG*LAGr9). The hydrophobic PCL segment forms the micellar core, while hydrophilic PEG constitutes the shell of the micelles. The peptide, PLG*LAG, has a specific cleavage site “*” for decladding the PEG layer from the micelleplex in tumors (Figure 4B). The cationic peptide polyarginine (R9) provides siRNA binding function and cellular membrane penetration. Micelleplexes based on such MMP-2- responsive nanoparticles are capable of enhancing siRNA delivery to tumor cells in vitro and in vivo and can efficiently inhibit breast cancer growth by delivering siRNA against Polo-like kinase 1 .

Other MMP cleavable peptides were also utilized as linkers to create sheddable PEG layers, such as PPDMEND (constructed with PEG/matrix metalloproteinase (MMP)-substrate peptide 20 /1,2-dioleoyl-sn-glycero-3-phosphoethanolamine (DOPE) ternary conjugate (PPD)), ${ }^{63}$ MMP-sensitive PAT element (poly[(DMAEMA)-
b-(DMAEMA-co-PAA-co-BMA)] $)^{56}$ and its upgraded version (FA ligands were utilized to enhance the cellular uptake instead of the zeta potential shift, Figure 4C). These studies indicate that these MMP-responsive delivery platforms offer significant potential for environmentally targeted, tissuespecific siRNA delivery.

\section{TME Hypoxia-Responsive Nanocarrier}

Due to rapid growth of solid tumors, the dense extracellular matrix and the vascular dysfunction in the tumor region, the majority of solid tumors develop acute hypoxia (less than $1.4 \%$ oxygen) in the TME. ${ }^{39}$ It has been widely accepted that hypoxia is related to tumorigenesis and therapy resistance. ${ }^{64,65}$ This scenario also provides a new specific delivery strategy by targeting the hypoxic TME. Several hypoxia-responsive prodrugs have been reported which were mainly based on nitroimidazoles, azo-groups, ferrocene and disulfide bonds. ${ }^{66}$ Similar strategies have been used to design nanocarriers, for example the azo linker was introduced to construct a hypoxia-responsive sheddable PEG layer ${ }^{67}$ or hypoxia degradable polymer. ${ }^{68}$ 4-nitrobenzyl chloroformate (NBCF)-modified side chains were also utilized to construct a hypoxia-responsive charge shiftable polymer (the charge of the nanocarrier was neutral in circulation, and the charge became positive upon reaching the hypoxic TME). ${ }^{45,69}$ However, the majority of nanocarriers are designed for drug delivery and due to the instability and difficulty of encapsulating the siRNA, it is relatively difficult to prepare a hypoxia-responsive nanocarrier for siRNA delivery as compared with drug delivery.

The first reported hypoxia-responsive nanocarrier for siRNA delivery was the hypoxia-sensitive polymer PAPD. The PEI-DOPE conjugate was utilized to form siRNA complexation and promote formation of micellar nanoparticles, and to achieve the hypoxia-responsive ability, an azobenzene linker was introduced. When the nanocarrier arrived at the TME, the azobenzene linker would be degraded, and as a result, the positive charge of PEI would be presented, leading to the cellular uptake of the PEI- DOPE/siRNA complexes. ${ }^{41}$ This research sheds light on the possibility of utilizing hypoxia as a TME-specific target for siRNA delivery.

\section{TME Redox-Responsive Nanocarrier}

The redox environments are quite distinct between the cytoplasm and extracellular space, which provides an opportunity for the design of multistage responsive nanocarriers. For 
example, the concentration of glutathione (GSH) in the cytoplasm is approximately $2-10 \times 10^{-3} \mathrm{M}$, while it decreases to $2-10 \times 10^{-6} \mathrm{M}$ in the extracellular fluid. ${ }^{58}$

The most commonly used redox-responsive functional group is the disulfide bonds which could be cleaved by GSH. Our group developed a disulfide bond-containing polymer (Poly (disulfide amide) (PDSA)). It was composed of a lipid-poly(ethylene glycol) shell and the PDSA/cationic lipid core which encapsulated the siRNA (Figure 5A). Once it reached the vicinity of tumors via the EPR effect and was internalized by tumor cells, the disulfide bonds in PDSA would be rapidly cleaved by the enriched GSH present in the cytoplasm, leading to the degradation of the nanocarrier and as a result, the release of the encapsulated cationic lipid/ siRNA complex. Due to the proton sponge effect induced by the positively charged cationic lipid/siRNA complex, the encapsulated siRNA could finally escape from the endosome with subsequent silencing of the targeted gene. ${ }^{58}$

Gao et al reported dual redox/pH responsive nanocarriers constructed with methoxy-poly (ethylene glycol)-polylactide -polyhistidine-ss-oligoethylenimine (mPEGb-PLA-Phis-ssOEI)-based polyplex, which exhibited an enhanced endo/ lysosomal escape ability (Figure 5B). The polyplex was designed to be a union between $\mathrm{pH}$-sensitive PEG-b-PLA-
PHis and a redox cleavable disulfide bond linked by OEI, where this design provided two responsive sites to achieve efficient multistage responsiveness. ${ }^{70}$

Several studies also reported a nanocarrier constructed by the conjugation of redox-responsive glucolipids (CSOss-SA) utilized for the co-delivery of siRNA and a small molecular drug. CSO-ss-SA demonstrated the ability to both condense the siRNA and form the nanocarriers, therefore offering the possibility for CSO-ss-SA to be utilized as a siRNA delivery vector. ${ }^{71}$

\section{Conclusion and Future Outlook}

This review has considered the barriers for siRNA delivery and the current solutions offered by nanocarriers. As illustrated in the beginning, siRNA suffers from first pass elimination, where more than $95 \%$ of siRNAs within the circulation would be eliminated by the liver, renal and RNases in the circulation during the few initial minutes. Even if the nanocarriers could arrive at the tumor region, the abundant negative charge of siRNAs would inhibit its cellular uptake. These barriers are the most important limitations plaguing the clinical application of RNAi technology.

To overcome these obstacles, a multistage nanodelivery system was invented. By utilizing specific stimuli

A
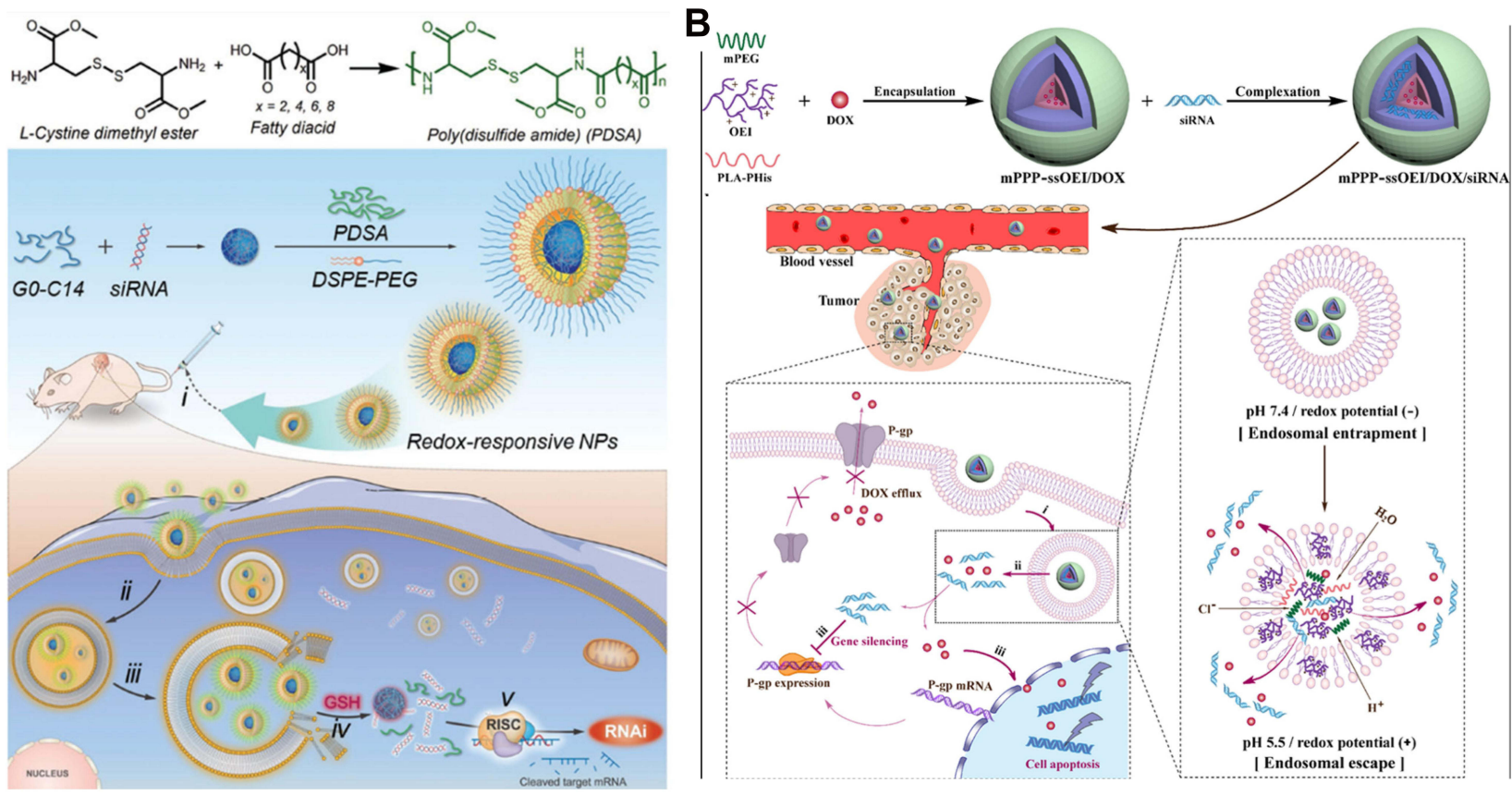

Figure 5 (A) The co-assembly of PDSA polymer with cationic lipid (G0-CI4), siRNA, and DSPE-PEG to form stable NPs with an embedded hydrophobic PDSA core with DSPE-PEG on the surface of the NPs; Reproduced from Xu X, Wu J, Liu S, et al. Redox-responsive nanoparticle-mediated systemic RNAi for effective cancer therapy. Small. 2018; 14:e 1802565 . Copyright 2018 John Wiley and Sons. ${ }^{58}$ (B) Co-assembly of mPEG, OEl and PLA-PHis encapsulating Dox to formulate a mPPP-ssOEI/Dox/siRNA delivery system; Reproduced from Gao Y, Wang $\mathrm{Q}$, Hu H, et al. pH/redox dual-responsive polyplex with effective endosomal escape for codelivery of siRNA and doxorubicin against drug-resistant cancer cells. ACS Appl Mater Interfaces. 2019;11:16,296-16,310. Copyright 2019 American Chemical Society. ${ }^{70}$ 
responsive elements combined with a hydrophilic PEG surface layer, the multistage delivery system would be able to achieve both stabilization in circulation and enhanced cellular uptake in the TME. The most commonly targeted stimuli in the TME are the acidic $\mathrm{pH}$, elevated enzymes, hypoxia and altered redox conditions. By introducing certain elements responsive to TME stimuli, the nanocarriers could change their physical or chemical characteristics (such as shape, charge, dis-PEGylation or disassembly) and rapidly release the encapsulated cationic/ siRNA complex upon reaching the TME. For example, the amide bond is frequently used as the TME acidic $\mathrm{pH}$ responsive element, MMP cleavable peptide is usually used as the element responsive to the enzymes present in the TME, azo group is typically used as the hypoxia responsive element, while the disulfide bond is most commonly used as the redox responsive element.

During recent years, more and more studies are focusing on the development of multistage nanocarriers, especially regarding exclusive siRNA delivery or combination delivery. Due to the benefits offered by these multistage nanocarriers such as the ability of precise tumor targeting and high efficiency of siRNA delivery, increasing numbers of "untargetable" proteins which are crucial in tumorigenesis are now "targetable". Therefore, it can be projected that the multistage siRNA delivery system would provide enormous potential for cancer therapy in the future.

\section{Abbreviation}

Ago2, Argonaute 2; CDM, Cyclohexanedimethanol; CSOss-SA, Redox-responsive glucolipids; DDS, Drug delivery system; DET, PEG-GPLGVRG-PAsp; DMMA, 2,3-Dimethylmaleic anhydride; DOPE, Phosphoethanolamine; dsRNA, Double-strand RNA; EPR, Enhanced permeability and retention; FDA, US Food and Drug Administration; GSH, Glutathione; hATTR, Hereditary transthyretin amyloidosis; MMPs, Matrix metalloproteinases; NBCF, Nitrobenzyl chloroformate; NgBR, Nogo-B receptor; NPs, Nanoparticles; PDSA, Polymer (Poly (disulfide amide); PEG, Polyethylene glycol; PEI, Polyethylene imine; PHMEMA, Hydrophobic poly(2-(hexamethyleneimino) ethyl methacrylate); PPD, PEG-Peptide-DOPE conjugate; PPP-NPs, PPP-modified PLGA nanoparticles; RISC, RNA-induced silencing complex; RNAi, RNA interference; ROS, Reactive oxygen species; siRNA, Small interfering RNA; S-NP, Sheddable nanoparticle; SOD, Superoxide dismutase; TME, Tumor microenvironment.

\section{Acknowledgments}

This work was supported by the National Natural Science Foundation of China (81874226, 81803020, 82050410363, 82072930), the International Scientific and Technological Cooperation Program from Guangdong Science and Technology Department (2018A050506033), the Hundred Talents Program for Young Scholars of Sun Yat-sen University (1320318003) the grant from Guangzhou Science and Technology Bureau (201704020131), the Guangdong Province Outstanding Youth Award (2021B1515020066), The Three Million for Three Years Project of SYSMH (132090023), and grant from Guangdong Science and Technology Department (2017B030314026).

\section{Disclosure}

The authors declare no conflict of interest.

\section{References}

1. Fire $\mathrm{A}, \mathrm{Xu} \mathrm{S}$, Montgomery $\mathrm{MK}$, et al. Potent and specific genetic interference by double-stranded RNA in caenorhabditis elegans. Nature. 1998;391(6669):806-811. doi:10.1038/35888

2. Elbashir SM, Harborth J, Lendeckel W, et al. Duplexes of 21-nucleotide RNAs mediate RNA interference in cultured mammalian cells. Nature. 2001;411(6836):494-498. doi:10.1038/35078107

3. Hannon GJ. RNA interference. Nature. 2002;418(6894):244-251. doi:10.1038/418244a

4. Crooke ST, Witztum JL, Bennett CF, Baker BF. RNA-targeted therapeutics. Cell Metab. 2018;27(4):714-739. doi:10.1016/j. cmet.2018.03.004

5. Hu B, Weng Y, Xia X-H, Liang X-J, Huang Y. Clinical advances of siRNA therapeutics. J Gene Med. 2019;21(7):e3097. doi:10.1002/ jgm.3097

6. Huang Y, Cheng Q, Ji J-L, et al. Pharmacokinetic behaviors of intravenously administered siRNA in glandular tissues. Theranostics. 2016;6(10):1528-1541. doi:10.7150/thno.15246

7. Tai W, Gao X. Noncovalent tagging of siRNA with steroids for transmembrane delivery. Biomaterials. 2018;178:720-727. doi:10.1016/j.biomaterials.2018.02.007

8. Zheng Y, Tai W. Insight into the siRNA transmembrane deliveryfrom cholesterol conjugating to tagging. Wiley Interdiscip Rev Nanomed Nanobiotechnol. 2020;12(3):e1606. doi:10.1002/ wnan. 1606

9. Jabir MS, Nayef UM, Abdulkadhim WK, et al. Fe3O4 nanoparticles capped with PEG induce apoptosis in breast cancer AMJ13 cells via mitochondrial damage and reduction of NF- $\mathrm{kB}$ translocation. J Inorg Organomet Polym Mater. 2021;31(3):1241-1259. doi:10.1007/ s10904-020-01791-4

10. Sharafaldin Al-Musawi SA, Al-Karagoly H, Sulaiman GM, Jabir MS, Naderi-Manesh H. Dextran-coated superparamagnetic nanoparticles modified with folate for targeted drug delivery of camptothecin. $A d v$ Nat Sci. 2020;11:045009. doi:10.1088/2043-6254/abc75b

11. Bader JE, Voss K, Rathmell JC. Targeting metabolism to improve the tumor microenvironment for cancer immunotherapy. Mol Cell. 2020;78(6):1019-1033. doi:10.1016/j.molcel.2020.05.034

12. Liu Y, Qiao L, Zhang S, et al. Dual $\mathrm{pH}$-responsive multifunctional nanoparticles for targeted treatment of breast cancer by combining immunotherapy and chemotherapy. Acta Biomater. 2018;66:310-324. doi:10.1016/j.actbio.2017.11.010 
13. Li Y, Ding J, Xu X, et al. Dual hypoxia-targeting RNAi nanomedicine for precision cancer therapy. Nano Lett. 2020;20(7):4857-4863. doi:10.1021/acs.nanolett.0c00757

14. Patil P, Gao YG, Lin X, et al. The development of functional non-viral vectors for gene delivery. Int $J$ Mol Sci. 2019;20 (21):5491. doi:10.3390/ijms20215491

15. Kareem SH, Naji AM, Taqi ZJ, Jabir MS. Polyvinylpyrrolidone loaded-MnZnFe2O4 magnetic nanocomposites induce apoptosis in cancer cells through mitochondrial damage and P53 pathway. $J$ Inorg Organomet Polym Mater. 2020;30(12):5009-5023. doi:10.1007/s10904-020-01651-1

16. Yin H, Kanasty RL, Eltoukhy AA, et al. Non-viral vectors for gene-based therapy. Nat Rev Genet. 2014;15(8):541-555. doi: $10.1038 / \mathrm{nrg} 3763$

17. Shi Y, van der Meel R, Chen X, Lammers T. The EPR effect and beyond: strategies to improve tumor targeting and cancer nanomedicine treatment efficacy. Theranostics. 2020;10(17):7921-7924. doi:10.7150/thno.49577

18. Hinshaw DC, Shevde LA. The tumor microenvironment innately modulates cancer progression. Cancer Res. 2019;79(18):4557-4566. doi:10.1158/0008-5472.CAN-18-3962

19. Fridman WH, Miller I, Sautes-Fridman C, Byrne AT. Therapeutic targeting of the colorectal tumor stroma. Gastroenterology. 2020;158 (2):303-321. doi:10.1053/j.gastro.2019.09.045

20. Arneth B. Tumor microenvironment. Medicina. 2019;56:15. doi:10.3390/medicina56010015

21. Tabernero J, Shapiro GI, LoRusso PM, et al. First-in-humans trial of an RNA interference therapeutic targeting VEGF and KSP in cancer patients with liver involvement. Cancer Discov. 2013;3(4):406-417. doi:10.1158/2159-8290.CD-12-0429

22. Cervantes A, Alsina M, Tabernero J, et al. Phase I dose-escalation study of ALN-VSP02, a novel RNAi therapeutic for solid tumors with liver involvement. J Clin Oncol. 2011;29(15_suppl):3025. doi:10.1200/jco.2011.29.15_suppl.3025

23. Davis ME, Zuckerman JE, Choi CHJ, et al. Evidence of RNAi in humans from systemically administered siRNA via targeted nanoparticles. Nature. 2010;464(7291):1067-1070. doi:10.1038/ nature 08956

24. Davis ME. The first targeted delivery of siRNA in humans via a self-assembling, cyclodextrin polymer-based nanoparticle: from concept to clinic. Mol Pharm. 2009;6(3):659-668. doi:10.1021/ $\mathrm{mp} 900015 \mathrm{y}$

25. Golan T, Khvalevsky EZ, Hubert A, et al. RNAi therapy targeting KRAS in combination with chemotherapy for locally advanced pancreatic cancer patients. Oncotarget. 2015;6(27):24560-24570. doi:10.18632/oncotarget.4183

26. Northfelt DW, Hamburg SI, Borad MJ, et al. A phase I dose-escalation study of TKM-080301, a RNAi therapeutic directed against polo-like kinase 1 (PLK1), in patients with advanced solid tumors: expansion cohort evaluation of biopsy samples for evidence of pharmacodynamic effects of PLK1 inhibition. J Clin Oncol. 2013;31(15_suppl):TPS2621-TPS2621.

doi: $10.1200 /$ jco.2013.31.15_suppl.tps2621

27. Semple SC, Judge AD, Robbins M, et al. Abstract 2829: preclinical characterization of TKM-080301, a lipid nanoparticle formulation of a small interfering RNA directed against polo-like kinase 1. Cancer Res. 2011;71:2829. doi:10.1158/1538-7445.am2011-2829

28. Tolcher AW, Papadopoulos KP, Patnaik A, et al. Safety and activity of DCR-MYC, a first-in-class Dicer-substrate small interfering RNA (DsiRNA) targeting MYC, in a phase I study in patients with advanced solid tumors. J Clin Oncol. 2015;33(15_suppl):11006. doi:10.1200/jco.2015.33.15_suppl.11006

29. Chipumuro E, Siddiquee Z, Ganesh S, et al. Abstract 2925: anti-tumor activity of a MYC-targeting dicer substrate siRNA in combination with BRD4/CDK7 inhibitors. Cancer Res. 2016;76:2925. doi:10.1158/1538-7445.am2016-2925
30. Landen CN Jr, Chavez-Reyes A, Bucana C, et al. Therapeutic EphA2 gene targeting in vivo using neutral liposomal small interfering RNA delivery. Cancer Res. 2005;65(15):6910-6918. doi:10.1158/00085472.CAN-05-0530

31. Bailey KM, Wojtkowiak JW, Cornnell HH, et al. Mechanisms of buffer therapy resistance. Neoplasia. 2014;16(4):354. doi:10.1016/j. neo.2014.04.005

32. de la Rica R, Aili D, Stevens MM. Enzyme-responsive nanoparticles for drug release and diagnostics. Adv Drug Deliv Rev. 2012;64 (11):967-978. doi:10.1016/j.addr.2012.01.002

33. Uthaman S, Huh KM, Park I-K. Tumor microenvironment-responsive nanoparticles for cancer theragnostic applications. Biomater Res. 2018;22(1):22. doi:10.1186/s40824-018-0132-z

34. Xu X, Saw PE, Tao W, et al. Tumor microenvironment-responsive multistaged nanoplatform for systemic RNAi and cancer therapy. Nano Lett. 2017;17(7):4427-4435. doi:10.1021/acs.nanolett.7b01571

35. Xu C-F, Zhang H-B, Sun C-Y, et al. Tumor acidity-sensitive linkage-bridged block copolymer for therapeutic siRNA delivery. Biomaterials. 2016;88:48-59. doi:10.1016/j. biomaterials.2016.02.031

36. Chen B, Dai W, He B, et al. Current multistage drug delivery systems based on the tumor microenvironment. Theranostics. 2017;7 (3):538-558. doi:10.7150/thno.16684

37. Egeblad M, Werb Z. New functions for the matrix metalloproteinases in cancer progression. Nat Rev Cancer. 2002;2(3):161-174. doi: $10.1038 / \mathrm{nrc} 745$

38. Kessenbrock K, Plaks V, Werb Z. Matrix metalloproteinases: regulators of the tumor microenvironment. Cell. 2010;141(1):52-67. doi:10.1016/j.cell.2010.03.015

39. Wilson WR, Hay MP. Targeting hypoxia in cancer therapy. Nat Rev Cancer. 2011;11(6):393-410. doi:10.1038/nrc3064

40. Thambi T, Deepagan VG, Yoon HY, et al. Hypoxia-responsive polymeric nanoparticles for tumor-targeted drug delivery. Biomaterials. 2014;35(5):1735-1743. doi:10.1016/j.biomaterials.2013.11.022

41. Perche F, Biswas S, Wang T, Zhu L, Torchilin VP. Hypoxia-targeted siRNA delivery. Angew Chem. 2014;53(13):3362-3366. doi:10.1002/ anie. 201308368

42. Wang L, Huo M, Chen Y, Shi SJ. Tumor microenvironment-enabled nanotherapy. Adv Healthcare Mater. 2018;7(8):e1701156. doi:10.1002/adhm.201701156

43. Ran Mo R, Gu Z. Tumor microenvironment and intracellular signal-activated nanomaterials for anticancer drug delivery. Mater Today. 2016;19(5):274-283. doi:10.1016/j.mattod.2015.11.025

44. Rosenblum D, Joshi N, Tao W, Karp JM, Peer D. Progress and challenges towards targeted delivery of cancer therapeutics. Nat Commun. 2018;9(1):1410. doi:10.1038/s41467-018-03705-y

45. Ge Z, Liu S. Functional block copolymer assemblies responsive to tumor and intracellular microenvironments for site-specific drug delivery and enhanced imaging performance. Chem Soc Rev. 2013;42(17):7289-7325. doi:10.1039/c3cs60048c

46. Saw PE, Song E-W. siRNA therapeutics: a clinical reality. Sci China Life Sci. 2020;63(4):485-500. doi:10.1007/s11427-018-9438-y

47. Cao S, Lin C, Liang S, et al. Enhancing chemotherapy by RNA interference. BIO Integr. 2020;1(2):64-81. doi:10.15212/bioi-20200003

48. Yang X-Z, Du J-Z, Dou S, et al. Sheddable ternary nanoparticles for tumor acidity-targeted siRNA delivery. ACS Nano. 2012;6 (1):771-781. doi: $10.1021 / \mathrm{nn} 204240 \mathrm{~b}$

49. Sun C-Y, Shen S, Xu C-F, et al. Tumor acidity-sensitive polymeric vector for active targeted siRNA delivery. J Am Chem Soc. 2015;137 (48):15217-15224. doi:10.1021/jacs.5b09602

50. Fan B, Kang L, Chen L, et al. Systemic siRNA delivery with a dual pH-responsive and tumor-targeted nanovector for inhibiting tumor growth and spontaneous metastasis in orthotopic murine model of breast carcinoma. Theranostics. 2017;7(2):357-376. doi:10.7150/ thno. 16855 
51. Wang Y, Xiao H, Fang J, et al. Construction of negatively charged and environment-sensitive nanomedicine for tumor-targeted efficient siRNA delivery. Chem Commun. 2016;52(6):1194-1197. doi:10.1039/c5cc09181k

52. Li J, Yu X, Wang Y, et al. A reduction and $\mathrm{pH}$ dual-sensitive polymeric vector for long-circulating and tumor-targeted siRNA delivery. Adv Mater. 2014;26(48):8217-8224. doi:10.1002/adma.201403877

53. Yang Y, Xie X, Yang Y, et al. Polymer nanoparticles modified with photo- and $\mathrm{pH}$-dual-responsive polypeptides for enhanced and targeted cancer therapy. Mol Pharm. 2016;13(5):1508-1519. doi:10.1021/acs.molpharmaceut.5b00977

54. Li J, Ge Z, Liu S. PEG-sheddable polyplex micelles as smart gene carriers based on MMP-cleavable peptide-linked block copolymers. Chem Commun (Camb). 2013;49(62):6974-6976. doi:10.1039/ c3cc43576h

55. Hatakeyama H, Akita H, Ito E, et al. Systemic delivery of siRNA to tumors using a lipid nanoparticle containing a tumor-specific cleavable PEG-lipid. Biomaterials. 2011;32(18):4306-4316. doi:10.1016/j. biomaterials.2011.02.045

56. Li H, Yu SS, Miteva M, et al. Matrix metalloproteinase responsive, proximity-activated polymeric nanoparticles for siRNA DELIVERY. Adv Funct Mater. 2013;23(24):3040-3052. doi:10.1002/ adfm.201202215

57. Li H, Miteva M, Kirkbride KC, et al. Dual MMP7-proximityactivated and folate receptor-targeted nanoparticles for siRNA delivery. Biomacromolecules. 2015;16(1):192-201. doi:10.1021/ bm501394m

58. Xu X, Wu J, Liu S, et al. Redox-responsive nanoparticle-mediated systemic RNAi for effective cancer therapy. Small. 2018;14(41): e1802565. doi:10.1002/smll.201802565

59. Wang B, Ding Y, Zhao X, et al. Delivery of small interfering RNA against Nogo-B receptor via tumor-acidity responsive nanoparticles for Tumor vessel normalization and metastasis suppression. Biomaterials. $\quad 2018 ; 175: 110-122$ doi:10.1016/j. biomaterials.2018.05.034

60. Chen J, Li X, Sun Y, et al. Synthesis of size-tunable hollow polypyrrole nanostructures and their assembly into folate-targeting and pH-responsive anticancer drug-delivery agents. Chemistry. 2017;23 (68):17279-17289. doi:10.1002/chem.201702945

61. Najafi M, Farhood B, Mortezaee K. Extracellular matrix (ECM) stiffness and degradation as cancer drivers. $J$ Cell Biochem. 2019;120:2782-2790. doi:10.1002/jcb.27681
62. Wang H-X, Yang X-Z, Sun C-Y, et al. Matrix metalloproteinase 2-responsive micelle for siRNA delivery. Biomaterials. 2014;35 (26):7622-7634. doi:10.1016/j.biomaterials.2014.05.050

63. Hatakeyama H, Akita H, Kogure K, et al. Development of a novel systemic gene delivery system for cancer therapy with a tumor-specific cleavable PEG-lipid. Gene Ther. 2007;14(1):68-77. doi:10.1038/sj.gt.3302843

64. Brown JM, Wilson WR. Exploiting tumour hypoxia in cancer treatment. Nat Rev Cancer. 2004;4(6):437-447. doi:10.1038/nrc1367

65. Rezaeian A-H, Li C-F, Wu C-Y, et al. A hypoxia-responsive TRAF6-ATM-H2AX signalling axis promotes HIF1alpha activation, tumorigenesis and metastasis. Nat Cell Biol. 2017;19(1):38-51. doi:10.1038/ncb3445

66. Zeng Y, Ma J, Zhan Y, et al. Hypoxia-activated prodrugs and redox-responsive nanocarriers. Int $J$ Nanomedicine. 2018;13:6551-6574. doi:10.2147/IJN.S173431

67. Im S, Lee J, Park D, et al. Hypoxia-triggered transforming immunomodulator for cancer immunotherapy via photodynamically enhanced antigen presentation of dendritic cell. ACS Nano. 2019;13 (1):476-488. doi:10.1021/acsnano.8b07045

68. Zhang X, Wu M, Li J, et al. Light-enhanced hypoxia-response of conjugated polymer nanocarrier for successive synergistic photodynamic and chemo-therapy. ACS Appl Mater Interfaces. 2018;10 (26):21909-21919. doi:10.1021/acsami.8b06491

69. Zhen J, Tian S, Liu Q, et al. Nanocarriers responsive to a hypoxia gradient facilitate enhanced tumor penetration and improved anti-tumor efficacy. Biomater Sci. 2019;7(7):2986-2995. doi:10.1039/c9bm00461k

70. Gao Y, Jia L, Wang Q, et al. pH/redox dual-responsive polyplex with effective endosomal escape for codelivery of siRNA and doxorubicin against drug-resistant cancer cells. ACS Appl Mater Interfaces. 2019;11:16296-16310. doi:10.1021/acsami.9b02016

71. Meng T, Lu B, Shao S, et al. Sequential therapy with redox-responsive glucolipid nanocarrier separately delivering siRNA and doxorubicin to overcome multidrug resistance. Int $J \quad$ Pharm. 2017;534(1-2):368-377. doi:10.1016/j. ijpharm.2017.10.036
International Journal of Nanomedicine

\section{Publish your work in this journal}

The International Journal of Nanomedicine is an international, peerreviewed journal focusing on the application of nanotechnology in diagnostics, therapeutics, and drug delivery systems throughout the biomedical field. This journal is indexed on PubMed Central, MedLine, CAS, SciSearch ${ }^{\circledR}$, Current Contents ${ }^{\circledR} /$ Clinical Medicine,
Journal Citation Reports/Science Edition, EMBase, Scopus and the Elsevier Bibliographic databases. The manuscript management system is completely online and includes a very quick and fair peer-review system, which is all easy to use. Visit http://www.dovepress.com/ testimonials.php to read real quotes from published authors. 